\title{
Epidemiological and genetical differences between classical and atypical scrapie cases
}

\author{
Gesine LÜHKEN ${ }^{\mathrm{a} *}$, Anne BUSCHMANN $^{\mathrm{b}}$, Horst BRANDT ${ }^{\mathrm{a}}$, Martin EIDEN ${ }^{\mathrm{b}}$, \\ Martin H. GROSCHUP ${ }^{\mathrm{b}}$, Georg ERHARDT ${ }^{\mathrm{a}}$ \\ ${ }^{\text {a }}$ Department of Animal Breeding and Genetics, Justus-Liebig University of Giessen, \\ 35390 Giessen, Germany \\ ${ }^{\mathrm{b}}$ Friedrich-Loeffler Institute (FLI), Institute for Novel and Emerging Infectious Diseases, 17493 \\ Greifswald-Insel Riems, Germany
}

(Received 16 June 2006; accepted 13 September 2006)

\begin{abstract}
The aim of this study was to analyze the epidemiology and prion protein (PrP) genetics in scrapie-affected sheep flocks in Germany. For this purpose, 224 German scrapie cases in sheep diagnosed between January 2002 and February 2006 were classified as classical or atypical scrapie and the amino acids at codons 136, 141, 154 and 171 were determined. Likewise, representative numbers of flock mates were genotyped. Significant epidemiological differences were observed between classical and atypical scrapie cases in regard to the numbers of scrapie-affected sheep within a flock, the sizes of flocks with only a single scrapie-positive sheep or more than one scrapiepositive sheep and the age distribution of the scrapie-positive sheep. Sheep with the ARQ/ARQ genotype had by far the highest risk for acquiring classical scrapie, but the risk for atypical scrapie was the highest for sheep carrying phenylalanine $(F)$ at position $141\left(\mathrm{AF}_{141} \mathrm{RQ}\right)$ and/or the AHQ haplotype. However, atypical scrapie also occurred with a notable frequency in sheep with the PrP haplotypes ARR and/or ARQ in combination with Leucine at position $141\left(\mathrm{AL}_{141} \mathrm{RQ}\right)$. Furthermore, six atypical scrapie-positive sheep carried the PrP genotype ARR/ARR. The high proportion of sheep flocks affected by atypical scrapie underscores the importance of this scrapie type.
\end{abstract}

sheep / classical scrapie / atypical scrapie / epidemiology / PrP polymorphisms

\section{INTRODUCTION}

Like bovine spongiform encephalopathy (BSE) and human Creutzfeldt-Jakobdisease (CJD), ovine and caprine scrapie belongs to the group of transmissible spongiform encephalopathies (TSE). These fatal degenerative disorders of the central nervous system are characterized by the accumulation of an abnormal isoform $\left(\mathrm{PrP}^{\mathrm{sc}}\right)$ of the cellular prion protein

* Corresponding author:

Gesine.Luehken@agrar.uni-giessen.de
$\left(\operatorname{PrP}^{\mathrm{c}}\right)$. For more than 250 years, this infectious disease has been observed in European sheep flocks and was spread to many other countries of the world [11], causing economical losses but was never regarded as a risk factor for human health. On the contrary, there is strong evidence that BSE causes variant CJD in humans [5, 21]. The origin of BSE is still unclear. It may either have originated from spontaneously diseased cattle [15], or from scrapie infected sheep that were fed to cattle, allowing scrapie eventually to cross the species 
barrier [38, 39]. For those countries or regions in which ovine scrapie has become epidemic, conventional elimination efforts (culling of symptomatic sheep or of complete flocks) have spanned decades and in most cases have not been successful $[11,35]$. However, for more than ten years it is known that the susceptibility of sheep to natural and experimental scrapie is influenced by amino acid polymorphisms at the positions 136, 154 and 171 of the prion protein (e.g. [2, 17, 24]). Consequently, current regulations of the European Union aim to eradicate ovine scrapie from the member states by selecting for the PrP haplotype $A_{136} R_{154} R_{171}(A R R)$, regarded to be most resistant to scrapie, and to eliminate the other haplotypes, mainly VRQ, which is suspected to be most susceptible (e.g. $[1,8,14,37])$. This is implemented within scrapie-affected flocks by genotyping and the subsequent culling of susceptible sheep (Commission Regulation 1492/2004) and within sheep populations by breeding programs (Commission Decision 2003/100/EC).

In recent years, "atypical" scrapie cases have been identified $[3,7]$. The most frequently found atypical phenotype is designated Nor98 since it was initially discovered in Norway and subsequently found in other EU member states [9, 29, 32, 33]. In atypical scrapie cases, sheep carrying PrP haplotypes known to be less susceptible (AHQ) or resistant (ARR) to classical scrapie have been affected, while the VRQ haplotype seemed to confer at least partial resistance to atypical scrapie strains [3, 6, 10, 29, 30, 33].

In addition to the variations at codons 136, 154 and 171, further ovine $\operatorname{PrP}$ polymorphisms were described (reviewed in [18]). Among these, an amino acid substitution (L/F) at codon 141 showed an association with the susceptibility to Nor98 [30]. The $F_{141}$ allele has been shown to occur in combination with the alleles
$\mathrm{A}_{136}, \mathrm{R}_{154}$ and $\mathrm{Q}_{171}$, forming the PrP haplotype AFRQ [4, 23, 30].

It has been shown that Nor98 and other atypical cases can be efficiently transmitted to transgenic mice expressing sheep PrP [27]. No data are yet available on whether this disease is transmitted naturally within a sheep flock.

The aim of this study was to compare aspects of scrapie epidemiology and prion protein genetics between German sheep flocks affected with classical or atypical scrapie in order to assess their significance for the German sheep population and the scrapie resistance breeding programme.

\section{MATERIALS AND METHODS}

\subsection{Scrapie cases and samples}

German scrapie cases that were diagnosed from January 2002 to March 2006 were included in the study. This comprises brain or muscle tissue samples of 224 scrapie-positive sheep from 113 flocks. In 97 of these flocks, an average of 50 adult flock mates was blood-sampled $(n=4759)$. Flock mates of 16 cases were not included because these scrapie-affected sheep had only been brought into the flocks a few months or years before they had been identified as scrapie-positive or because there were no flock mates. According to Commission Regulation 1492/2004, depending on their PrP genotypes and on the ARR frequency within an affected flock, a proportion of the sampled flock mates had been culled and tested scrapie-negative by a TSE rapid test, while others were kept alive and remained free of clinical symptoms. Information on sex, age and breed of scrapie-affected sheep and their flock mates, on the manner of death of scrapieaffected sheep and on the geographical localization and size of the affected flocks was obtained from the responsible veterinary authorities, from the sheep keepers 
or during sample collection. When records were not available, the age of sheep was estimated by inspection of the teeth and was used to sample flock mates of approximately the same age as the scrapie-positive sheep. However, it was not possible to obtain all this information for all animals or flocks.

\subsection{Diagnosis of scrapie and scrapie type}

All TSE cases were initially identified using the Bio-Rad Platelia rapid test (Bio-Rad Laboratories, Hercules, California, USA, now Bio-Rad TeSeE test) and were confirmed by SAF-immunoblot and/or immunohistochemistry according to standardized methodology [31]. All TSE cases subsequently underwent a discriminatory testing [19] to verify the absence of BSE infection in these animals according to EU Regulation 36/2005. The cases were grouped into the classical or atypical scrapie categories on the basis of the immunoblotting patterns and glycotype profiles of the abnormal prion protein as well on the basis of its distinct proteinase $\mathrm{K}$ resistance and epitope accessibility characteristics [20].

\subsection{Genotyping}

DNA was extracted from brain or muscle tissue of scrapie-positive sheep with the QIAamp DNA Mini Kit (Qiagen, Hilden, Germany) and from peripheral blood leucocytes, tongue or ear tissue of flock-mates with the Invisorb Blood Mini HTS 96 Kit for sheep (Invitek, Berlin, Germany).

A $970 \mathrm{bp}$ fragment including the complete coding region of the $\operatorname{PrP}$ gene was amplified by PCR for all samples of scrapie-positive sheep, using the forward primer 5'-tgccactgctatacagtcatt-3' and the reverse primer 5'-aagcaagaaatgag acaccacc-3' with conventional PCR conditions. These PCR products were sequenced on both strands with PCR primers using an ABI PRISM 377 automated sequencer and Big Dye Terminator chemistry (Applied Biosystems, Foster City, USA). Polymorphisms at codons 136, 141, 154 and 171 were analyzed by visual inspection of the resulting chromatograms, supported by the software ChromasPro version 1.33 (Technelysium Pty Ltd, Tewantin, Australia) and Mutation Surveyor version 2.61 (Softgenetics, State College, USA), using the GenBank sequence U67922 as a reference sequence. In order to determine the PrP haplotypes constituted by codons 136 , 154 and 171, all samples of flock mates as well as samples of all scrapie-positive sheep that were heterozygous at more than one of these three codons were analyzed by the PCR-RFLP method as described by [28]. This method was optimized by using shorter primers (forward primer 5'-ggcaggagctgctgcagct-3'; reverse primer for digestion with $B s p \mathrm{HI} 5^{\prime}$ cacaaagttgttctggttactatc-3'; reverse primer for double digestion with $B s p \mathrm{HI}$ and $B s p \mathrm{DI}$ 5' -caaagttgttctggttactatat-3').

For determination of the genotype at codon 141 in the samples of the flock mates of scrapie-positive sheep and for verification of the genotype at codon 141 in sequenced samples of scrapiepositive sheep, a PCR-RFLP method was established using forward primer 5'acaagcccagtaagccaaaaacc-3' and reverse primer 5'-ctcatagtcattgccaaaaygttta-3' to amplify a 140 bp fragment by standard PCR conditions. Digestion with the restriction enzyme DraI resulted in fragments of 116 and 24 bp when $L$ was present at position 141.

Because of limited quantity or quality, molecular genetic analyses did not provide a result for some of the samples, therefore numbers of investigated sheep or flocks vary between the different analyses that were done. 


\subsection{Statistics}

Considering the different numbers of sampled flock mates in each scrapieaffected flock, the frequencies of haplotypes and genotypes regarding $\operatorname{PrP}$ codons 136, 154 and 171 were estimated for each scrapie-affected flock before calculating average frequencies over all flocks.

To prevent an influence of the PrP haplotypes regarding codons 136, 154 and 171 on the association analysis of the PrP codon 141 alleles with classical and atypical scrapie susceptibility, 177 pairs of scrapie-positive sheep and their flock mates with matching PrP genotypes with respect to codons 136, 154 and 171 were constituted.

The significance of differences between compared groups (e.g. classical scrapiepositive sheep and atypical positive sheep) in respect to epidemiological aspects (e.g. age at the time of scrapie diagnosis) or results of molecular genetic analyses (e.g. frequency of the $\mathrm{F}_{141}$ allele) was tested with the chi-square and Fisher exact test (in the case of low numbers of observations within subclasses).

The relative scrapie "risk" (odds ratio, OR) of a PrP haplotype or genotype was calculated for classical scrapie and for atypical scrapie as:

\footnotetext{
frequency $(\%)$ of haplotype or genotype in positive sheep / frequency $(\%)$ of haplotype or genotype in flock mates

frequency $(\%)$ of $\mathrm{ARQ}^{1}$ or $\mathrm{ARQ} / \mathrm{ARQ}^{1}$ in positive sheep / frequency $(\%)$ of $\mathrm{ARQ}^{1}$ or $\mathrm{ARQ} / \mathrm{ARQ}^{1}$ in flock mates

(1) $\mathrm{ARQ}$ or $\mathrm{ARQ} / \mathrm{ARQ}$ in combination with the $\mathrm{L}_{141}$ allele in analyses where the polymorphism at codon 141 was determined.
}

An OR higher than 1 means a higher susceptibility than the ARQ haplotype or the ARQ/ARQ genotype; an odds ratio lower than 1 means a susceptibility lower than the ARQ haplotype or the ARQ/ARQ genotype.

\section{RESULTS}

\subsection{Epidemiology}

All 224 scrapie-positive sheep included in this study were ewes, of which 120 were diagnosed with classical scrapie and 97 cases were diagnosed with atypical scrapie showing immunoblotting patterns reminiscent of Nor98. Inconclusive results for the scrapie type were obtained for 7 scrapie cases due to limited or unsuitable brain samples. Sheep affected with classical scrapie were identified in 16 flocks ("classical scrapie flocks") and sheep with atypical scrapie in 88 flocks ("atypical scrapie flocks") (Tab. I). Sheep affected with different scrapie types have not been identified within the same flock. However, positive sheep with inconclusive results originated from classical scrapie flocks and also from atypical scrapie flocks. In all flocks affected by classical scrapie, $1.7 \%$ of the sheep were classical scrapie-positive, while in atypical scrapie flocks, $0.2 \%$ of the sheep were diagnosed positive for atypical scrapie. In classical scrapie flocks, the number of identified positive sheep ranged from 1 to 57 (average: 7.5), compared to 1,2 or 3 positive sheep in atypical scrapie flocks (average: 1.1). There was a highly significant $(P<0.001)$ difference between classical scrapie flocks and atypical scrapie flocks regarding the identification of only a single or more than one scrapie-positive sheep. More than one sheep infected with classical scrapie was identified in more than $50 \%$ of the classical scrapie flocks, while only a single scrapiepositive sheep was found in more than $90 \%$ of the flocks where atypical scrapie was diagnosed (Tab. I).

Sheep affected with atypical scrapie were identified in all Federal German states except Schleswig-Holstein, Saarland and the small city states (Fig. 1). In contrast, sheep infected with classical scrapie were found in 
Table I. Numbers of scrapie-positive sheep and of scrapie-affected flocks with respect to the scrapie type.

\begin{tabular}{lcccccc}
\hline Scrapie-positive sheep per flock & \multicolumn{2}{c}{ Classical scrapie flocks } & & \multicolumn{2}{c}{ Atypical scrapie flocks } \\
\cline { 2 - 3 } \cline { 6 - 7 }$n$ & $n$ & $\%$ & & $n$ & $\%$ \\
\hline 1 & 7 & 43.8 & & 81 & 92.0 \\
2 & 2 & 12.5 & & 5 & 5.7 \\
3 & 1 & 6.3 & & 2 & 2.3 \\
4 & 1 & 6.3 & & - & - \\
5 & 1 & 6.3 & & - & - \\
8 & 1 & 6.3 & & - & - \\
15 & 1 & 6.3 & & - & - \\
17 & 1 & 6.3 & & - & - \\
57 & 1 & 6.3 & & - & - \\
Total & 1 & & $\mathbf{1 0 0 . 0}$ & & $\mathbf{8 8}$ & $\mathbf{1 0 0 . 0}$ \\
\hline
\end{tabular}

only 7 of the 16 Federal German states (Mecklenburg-Vorpommern, Brandenburg, Sachsen-Anhalt, Niedersachsen, Nordrhein-Westfalen, Thüringen and Baden-Württemberg). In other words, classical scrapie cases were - with one exception - not observed in southern Germany (Fig. 1).

In about $73 \%$ of all scrapie-affected flocks (classical and atypical), sheep were crossbred, and/or purebred sheep of different breeds were kept within the same flock. In total, purebred flocks of eleven different breeds were affected with classical and/or atypical scrapie, as listed in Table II. Not more than three purebred flocks of one breed were affected by scrapie, with the exception of the Merinolandschaf breed. In twelve purebred flocks of this breed, sheep affected with atypical scrapie were found, while no purebred Merinolandschaf flock was affected by classical scrapie.

The number of sheep in a flock at the time when the first scrapie-positive sheep was identified was recorded in 98 flocks. The flock sizes ranged from 1 to 5000 sheep, including lambs. The flock sizes were compared between flocks in which only one scrapie-positive sheep was identified and flocks in which more than one scrapie-positive sheep were identified (Tab. III). More than one sheep infected with classical scrapie was observed in flocks of all sizes between 1 and 1500 sheep, but the occurrence of more than one sheep affected with atypical scrapie was restricted to flocks with more than 500 animals (Tab. III). This difference in the flock sizes was highly significant $(P<0.001)$.

The age of 60 sheep with atypical and 20 sheep with classical scrapie at the time of death was recorded and was divided into five age groups (Tab. IV). Eighty percent of classical scrapie-positive sheep with known ages belonged to the age group of $3-5$ years. Two sheep (10\%) were younger than 3 years and two sheep were between 5 and 7 years of age. In contrast, atypical scrapie occurred in all five age groups. Almost $60 \%$ of the sheep affected with atypical scrapie with known age were older than 5 years, while more than a quarter was more than 10 years old (Tab. IV). There was a significant $(P<0.01)$ difference in the age distribution between sheep affected with classical and with atypical scrapie.

In 189 sheep where the type of scrapie could be determined, the manner of death is known (Tab. V). While $76.1 \%$ of 


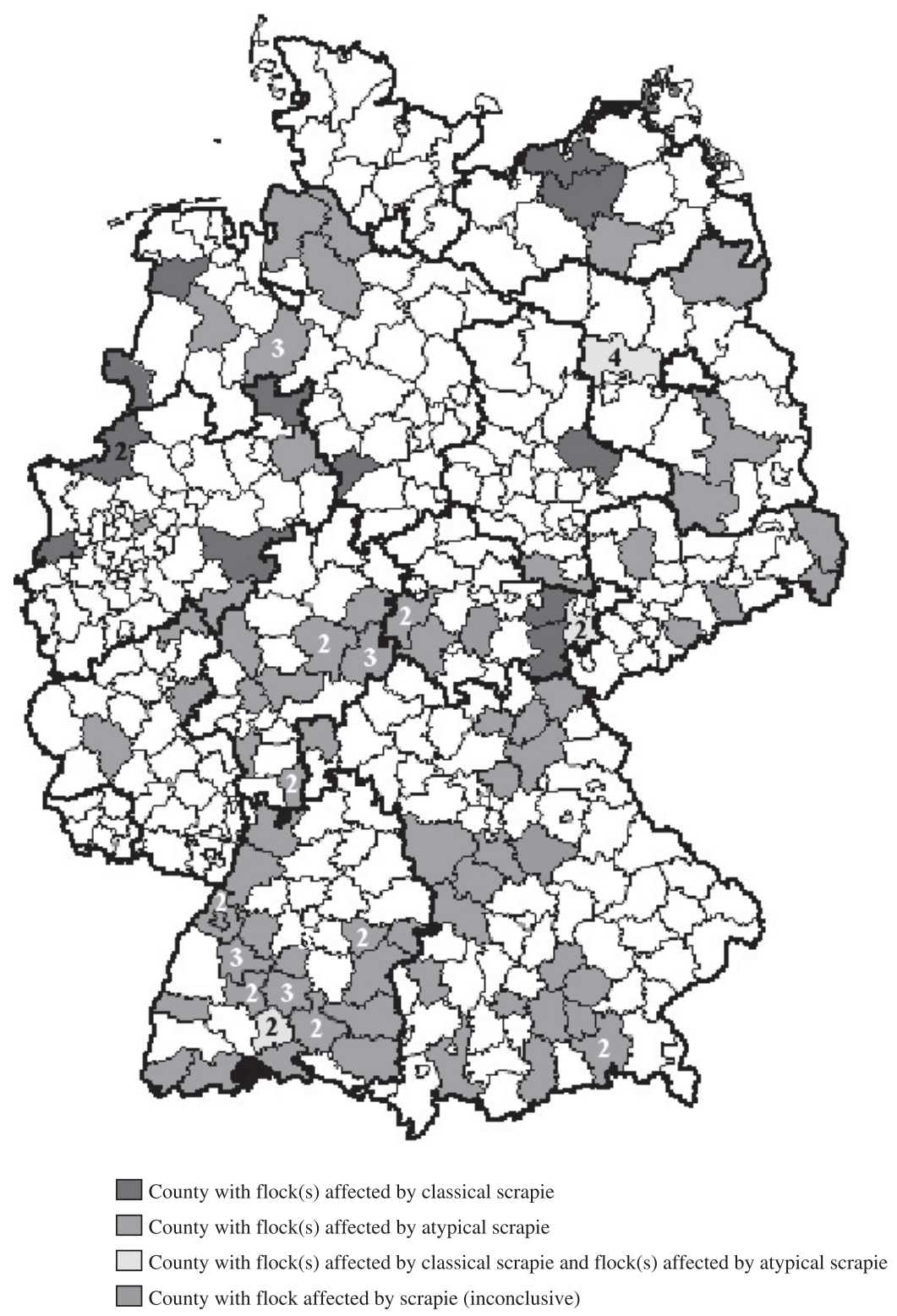

Figure 1. Regional distribution of German scrapie cases (January 2002-February 2006). Counties in which scrapie-affected flocks were identified are colored and numbers are given for more than a single scrapie-affected flock occurring in the same county. (A color version of this figure is available at www.edpsciences.org/vetres.) 
Table II. Breeds of scrapie-affected flocks with respect to the scrapie type.

\begin{tabular}{|c|c|c|c|c|}
\hline \multirow[t]{2}{*}{ Breed } & \multicolumn{2}{|c|}{ Classical scrapie flocks } & \multicolumn{2}{|c|}{ Atypical scrapie flocks } \\
\hline & $n$ & $\%$ & $n$ & $\%$ \\
\hline Coburger Fuchsschaf & - & - & 3 & 3.4 \\
\hline Deutsches Schwarzköpfiges Fleischschaf & - & - & 2 & 2.3 \\
\hline Graue Gehörnte Heidschnucke & - & - & 2 & 2.3 \\
\hline Merinolandschaf & - & - & 12 & 13.7 \\
\hline Merinolangwollschaf & - & - & 1 & 1.1 \\
\hline Moorschnucke & - & - & 1 & 1.1 \\
\hline Ostfriesisches Milchschaf & - & - & 1 & 1.1 \\
\hline Shropshire & 1 & 6.3 & 1 & 1.1 \\
\hline Suffolk & 1 & 6.3 & - & - \\
\hline Texel & 1 & 6.3 & 1 & 1.1 \\
\hline Weißes Bergschaf & - & - & 1 & 1.1 \\
\hline \multicolumn{5}{|l|}{ Crossbreed / more than one breed within } \\
\hline the same flock & 13 & 81.3 & 63 & 71.7 \\
\hline Total & 16 & 100.0 & 88 & 100.0 \\
\hline
\end{tabular}

Table III. Sizes of scrapie-affected flocks with respect to the number of scrapie-positive sheep and the scrapie type.

\begin{tabular}{|c|c|c|c|c|c|c|c|c|}
\hline \multirow{3}{*}{$\begin{array}{l}\text { Flock size } \\
(n)\end{array}$} & \multicolumn{4}{|c|}{ Classical scrapie flocks } & \multicolumn{4}{|c|}{ Atypical scrapie flocks } \\
\hline & \multicolumn{2}{|c|}{$\begin{array}{l}\text { with } 1 \text { scrapie- } \\
\text { positive sheep }\end{array}$} & \multicolumn{2}{|c|}{$\begin{array}{c}\text { with }>1 \text { scrapie- } \\
\text { positive sheep }\end{array}$} & \multicolumn{2}{|c|}{$\begin{array}{l}\text { with } 1 \text { scrapie- } \\
\text { positive sheep }\end{array}$} & \multicolumn{2}{|c|}{$\begin{array}{c}\text { with }>1 \text { scrapie- } \\
\text { positive sheep }\end{array}$} \\
\hline & $n$ & $\%$ & $n$ & $\%$ & $n$ & $\%$ & $\bar{n}$ & $\%$ \\
\hline $1-100$ & 6 & 85.7 & 1 & 12.5 & 36 & 47.4 & - & - \\
\hline $101-500$ & - & - & 2 & 25.0 & 13 & 17.1 & - & - \\
\hline $501-1000$ & - & - & 4 & 50.0 & 19 & 25.0 & 2 & 28.6 \\
\hline $1001-1500$ & 1 & 14.3 & 1 & 12.5 & 5 & 6.6 & - & - \\
\hline $1501-5000$ & - & - & - & - & 3 & 3.9 & 5 & 71.4 \\
\hline Total & 7 & 100.0 & 8 & 100.0 & 76 & 100.0 & 7 & 100.0 \\
\hline
\end{tabular}

classical scrapie-positive sheep were put to death during eradication culls in a scrapiepositive flock, no classical scrapie-affected sheep was diagnosed in a routinely slaughtered animal. However, about a quarter of the sheep diagnosed with atypical scrapie had been routinely slaughtered and twothirds of the sheep were fallen stock. The difference in the distribution of the man- ner of death between classical and atypical scrapie-positive sheep was highly significant $(P<0.001)$.

Central nervous symptoms were observed by veterinarians or sheep keepers in only a few cases. This has been reported for three sheep diagnosed with classical scrapie and nine sheep diagnosed with atypical scrapie. 
Table IV. Ages of scrapie-positive sheep with respect to the scrapie type.

\begin{tabular}{lccccc}
\hline $\begin{array}{l}\text { Age } \\
\text { (years) }\end{array}$ & \multicolumn{2}{c}{$\begin{array}{c}\text { Classical scrapie- } \\
\text { positive sheep }\end{array}$} & & \multicolumn{2}{c}{$\begin{array}{c}\text { Atypical scrapie- } \\
\text { positive sheep }\end{array}$} \\
\cline { 2 - 3 } \cline { 5 - 6 } & $n$ & $\%$ & & $n$ & $\%$ \\
\hline$<3$ & 2 & 10.0 & & 5 & 8.3 \\
$3-5$ & 16 & 80.0 & & 20 & 33.3 \\
$6-7$ & 2 & 10.0 & & 9 & 15.0 \\
$8-10$ & - & - & & 10 & 16.7 \\
$>10$ & - & - & & 16 & 26.7 \\
Total & $\mathbf{2 0}$ & $\mathbf{1 0 0 . 0}$ & & $\mathbf{6 0}$ & $\mathbf{1 0 0 . 0}$ \\
\hline
\end{tabular}

Table V. Manner of death of scrapie-positive sheep with respect to the scrapie type.

\begin{tabular}{lcccc}
\hline $\begin{array}{l}\text { Manner } \\
\text { of death }\end{array}$ & \multicolumn{2}{c}{$\begin{array}{c}\text { Classical scrapie- } \\
\text { positive sheep }\end{array}$} & \multicolumn{2}{c}{$\begin{array}{c}\text { Atypical scrapie- } \\
\text { positive sheep }\end{array}$} \\
\cline { 2 - 3 } \cline { 5 - 6 } & $n$ & $\%$ & & $\%$ \\
\hline Slaughtered & 0 & - & 23 & 27.3 \\
Fallen & 23 & 22.0 & 55 & 65.5 \\
Euthanised & 2 & 1.9 & 4 & 4.8 \\
Culled & 80 & 76.1 & 2 & 2.4 \\
Total & $\mathbf{1 0 5}$ & $\mathbf{1 0 0 . 0}$ & $\mathbf{8 4}$ & $\mathbf{1 0 0 . 0}$ \\
\hline
\end{tabular}

\subsection{Molecular genetics}

The frequencies of PrP haplotypes and genotypes of scrapie-positive sheep and their flock mates regarding codons 136, 154 and 171 are shown in Table VI. The distribution difference of haplotype frequencies was highly significant $(P<$ 0.001 ) between sheep affected with classical scrapie and their flock mates on the one hand and sheep affected with atypical scrapie and their flock mates on the other hand. The same was observed within the scrapie-positive sheep of each type and within the flock mates of scrapiepositive sheep of each type. In sheep affected with classical scrapie, the ARQ haplotype and the ARQ/ARQ genotype were by far the most dominant. Only the ARQ allele and its homozygous genotype were identified with higher frequencies in classical scrapie-positive sheep than in their flock mates. The PrP haplotypes AHQ, VRQ and ARH and their heterozygous genotypes were observed with a low frequency (less than 4\%) in classical scrapiepositive sheep while the according frequencies were slightly higher (or equal in the case of $A R H / V R Q)$ in their flock mates. Odds ratios (OR) of these haplotypes and genotypes were notably lower than 1 (Fig. 2). The ARR haplotype was not identified in the sheep diagnosed with classical scrapie, while the ARR frequency was about $29 \%$ in the flock mates.

In the group of sheep affected with atypical scrapie, the frequencies of the haplotypes ARQ, ARR, ARH and VRQ were lower than in their flock mates (Tab. VI), resulting in OR lower than 1 for ARR, ARH and VRQ (Fig. 2). Instead, the frequency of the AHQ haplotype was higher in atypical scrapie-positive sheep than in their flock mates with an OR of 3.24 (Fig. 2). The most frequent PrP genotype in sheep affected with atypical scrapie regarding codons 136, 154 and 171 was AHQ/ARQ (30.7\%), followed by ARQ/ARQ (25.0\%) and the ARR haplotype combined with ARQ (13.7\%) or AHQ (11.4\%). Again, OR were obviously higher than 1 only for genotypes with at least one AHQ haplotype, which were the genotypes AHQ/AHQ, AHQ/ARH, AHQ/ARQ and ARR/AHQ (Fig. 2). It has to be mentioned that six sheep $(6.8 \%)$ where atypical scrapie was diagnosed carried the ARR/ARR genotype and 28 (31.9\%) carried at least one ARR haplotype.

Due to the linkage of $F_{141}$ with the ARQ haplotype, all sheep homozygous for $F_{141}$ were also homozygous for the ARQ haplotype, and all $\mathrm{F}_{141}$ heterozygous animals carried at least one ARQ haplotype. The frequencies of the PrP haplotypes $\mathrm{AL}_{141} \mathrm{RQ}$ (ALRQ) and $\mathrm{AF}_{141} \mathrm{RQ}$ (AFRQ) and the resulting genotypes in scrapie- 
Table VI. PrP haplotype and genotype frequencies (codons 136, 154 and 171) in scrapie-positive sheep and their flock mates regarding the scrapie type.

\begin{tabular}{|c|c|c|c|c|c|c|c|c|}
\hline & \multicolumn{2}{|c|}{$\begin{array}{c}\text { Classical scrapie- } \\
\text { positive sheep }\end{array}$} & \multicolumn{2}{|c|}{$\begin{array}{l}\text { Flock mates of classical } \\
\text { scrapie-positive sheep }\end{array}$} & \multicolumn{2}{|c|}{$\begin{array}{c}\text { Atypical scrapie- } \\
\text { positive sheep } \\
\end{array}$} & \multicolumn{2}{|c|}{$\begin{array}{l}\text { Flock mates of atypical } \\
\text { scrapie-positive sheep }\end{array}$} \\
\hline & $n$ & $\%$ & $n$ & $\%$ & $n$ & $\%$ & $n$ & $\%$ \\
\hline \multicolumn{9}{|l|}{ Haplotype } \\
\hline AHQ & 4 & 1.7 & 94 & 6.3 & 56 & 31.8 & 873 & 10.9 \\
\hline ARH & 1 & 0.4 & 42 & 2.8 & 1 & 0.6 & 144 & 1.8 \\
\hline ARQ & 225 & 96.2 & 845 & 56.5 & 84 & 47.7 & 4343 & 54.2 \\
\hline ARR & - & - & 433 & 28.9 & 34 & 19.3 & 2500 & 31.2 \\
\hline VRQ & 4 & 1.7 & 82 & 5.5 & 1 & 0.6 & 144 & 1.8 \\
\hline ARK & - & - & - & - & - & - & 8 & 0.1 \\
\hline Total & 234 & 100.0 & 1496 & 100.0 & 176 & 100.0 & 8012 & 100.0 \\
\hline \multicolumn{9}{|l|}{ Genotype } \\
\hline AHQ/AHQ & - & - & 19 & 2.5 & 9 & 10.2 & 70 & 1.7 \\
\hline AHQ/ARH & - & - & 4 & 0.6 & 1 & 1.1 & 26 & 0.6 \\
\hline AHQ/ARQ & 4 & 3.4 & 41 & 5.5 & 27 & 30.7 & 484 & 12.1 \\
\hline ARH/VRQ & 1 & 0.9 & 7 & 0.9 & - & - & 3 & 0.1 \\
\hline ARQ/ARQ & 109 & 93.1 & 270 & 36.1 & 22 & 25.0 & 1284 & 32.1 \\
\hline ARQ/VRQ & 3 & 2.6 & 43 & 5.8 & 1 & 1.1 & 64 & 1.6 \\
\hline ARR/AHQ & - & - & 11 & 1.4 & 10 & 11.4 & 221 & 5.5 \\
\hline ARR/ARQ & - & - & 205 & 27.4 & 12 & 13.7 & 1166 & 29.1 \\
\hline ARR/ARR & - & - & 85 & 11.4 & 6 & 6.8 & 505 & 12.6 \\
\hline AHQ/VRQ & - & - & 1 & 0.1 & - & - & 5 & 0.1 \\
\hline ARH/ARH & - & - & - & - & - & - & 1 & 0.0 \\
\hline ARQ/ARH & - & - & 16 & 2.1 & - & - & 62 & 1.5 \\
\hline ARR/ARH & - & - & 15 & 2.0 & - & - & 47 & 1.2 \\
\hline ARR/VRQ & - & - & 31 & 4.2 & - & - & 57 & 1.4 \\
\hline VRQ/VRQ & - & - & - & - & - & - & 6 & 0.1 \\
\hline ARR/ARK & - & - & - & - & - & - & 1 & 0.0 \\
\hline ARH/ARK & - & - & - & - & - & - & 3 & 0.1 \\
\hline ARQ/ARK & - & - & - & - & - & - & 1 & 0.0 \\
\hline Total & 117 & 100.0 & 748 & 100.0 & 88 & 100.0 & 4006 & 100.0 \\
\hline
\end{tabular}

positive sheep and genotype (codons 136 , 154 and 171)-matched flock mates of each scrapie type are shown in Table VII. These frequencies were not significantly differently distributed between classical scrapie-positive sheep and their flock mates. In contrast, they differed sig- nificantly $(P<0.001)$ between the atypical scrapie-positive sheep and their flock mates. While $19.7 \%$ of atypical scrapiepositive sheep carried the AFRQ haplotype, its frequency was only $3.7 \%$ in their flock mates. A similar situation was observed for the genotypes including at least 


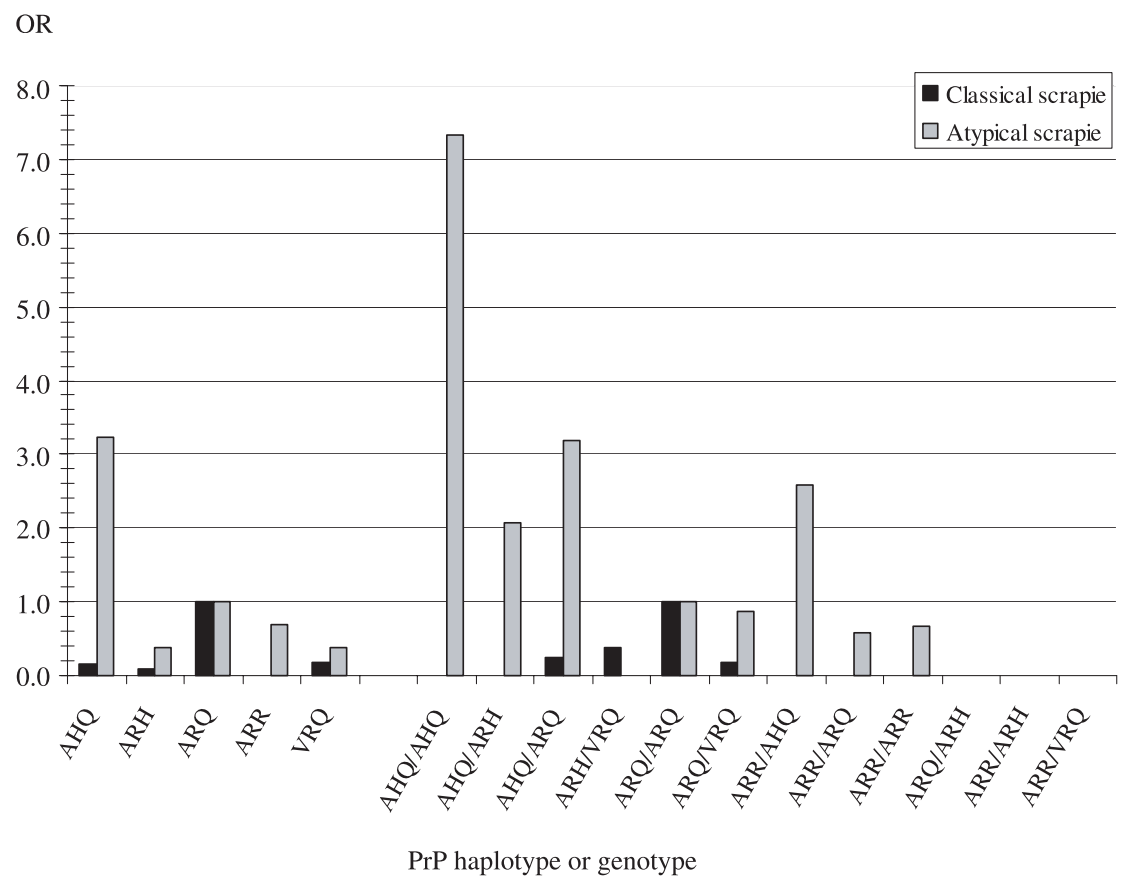

Figure 2. Odds ratios of PrP haplotypes and genotypes (codons 136, 154 and 171) in respect to the scrapie type. For each haplotype or genotype, the relative classical or atypical scrapie risk (odds ratio, OR) was calculated by dividing the quotient of its frequency (\%) in scrapie-positive sheep and its frequency $(\%)$ in the flock mates by the quotient of the ARQ or the ARQ/ARQ frequency $(\%)$ in scrapie-positive sheep and in the flock mates.

one AFRQ haplotype comparing sheep affected with atypical scrapie and their flock mates (Tab. VII). Interestingly, only few $(7.4 \%)$ of the atypical scrapie-positive sheep carried the ALRQ/ALRQ genotype, compared to a frequency of $21 \%$ within the genotype (codons 136, 154 and 171)matched flock mates.

The OR for the AFRQ haplotype (8.45) and the genotypes including one or two AFRQ haplotypes were remarkably high (between 9.49 and 22.86) for atypical scrapie, with the highest value for the combination of AFRQ with AHQ (Fig. 3). In contrast, among the PrP genotypes affected by atypical scrapie, ALRQ/ALRQ showed the lowest OR. For classical scrapie, OR for the AFRQ haplotype and the
AFRQ/ALRQ genotype were both lower than 1 (Fig. 3).

In both groups of sheep affected with classical or atypical scrapie, neither $\operatorname{PrP}$ haplotype frequencies nor PrP genotype frequencies differed significantly in their distribution within the different age groups.

\section{DISCUSSION}

One of the most striking aspects of atypical scrapie is that only a single scrapie-positive sheep per affected flock was identified in most cases $[9,30]$. This is one aspect which led to the speculation that atypical scrapie may be a spontaneous disease like sporadic Creutzfeldt-Jakob disease (CJD) in humans [3,30]. 
Table VII. Frequencies of PrP haplotypes $\mathrm{AL}_{141}$ RQ (ALRQ) and $\mathrm{AF}_{141} \mathrm{RQ}$ (AFRQ) and of the resulting genotypes of pairs of scrapie-positive sheep and flock mates with matched PrP genotypes (in respect to codons 136, 154 and 171) regarding the scrapie type.

\begin{tabular}{|c|c|c|c|c|c|c|c|c|}
\hline & \multicolumn{2}{|c|}{$\begin{array}{c}\text { Classical scrapie- } \\
\text { positive sheep }\end{array}$} & \multicolumn{2}{|c|}{$\begin{array}{l}\text { Flock mates of classical } \\
\text { scrapie-positive sheep }\end{array}$} & \multicolumn{2}{|c|}{$\begin{array}{l}\text { Atypical scrapie- } \\
\text { positive sheep }\end{array}$} & \multicolumn{2}{|c|}{$\begin{array}{l}\text { Flock mates of atypical } \\
\text { scrapie-positive sheep }\end{array}$} \\
\hline & $n$ & $\%$ & $n$ & $\%$ & $n$ & $\%$ & $n$ & $\%$ \\
\hline \multicolumn{9}{|l|}{ Haplotype } \\
\hline ALRQ & 180 & 93.7 & 175 & 91.1 & 45 & 27.8 & 71 & 43.8 \\
\hline AFRQ & 4 & 2.1 & 9 & 4.7 & 32 & 19.7 & 6 & 3.7 \\
\hline Others ${ }^{\mathrm{a}}-\mathrm{L}_{141}$ & 8 & 4.2 & 8 & 4.2 & 85 & 52.5 & 85 & 52.5 \\
\hline Others $^{\mathrm{a}}-\mathbf{F}_{141}$ & - & - & - & - & - & - & - & - \\
\hline Total & 192 & 100.0 & 192 & 100.0 & 162 & 100.0 & 162 & 100.0 \\
\hline \multicolumn{9}{|l|}{ Genotype $^{a}$} \\
\hline ALRQ/ALRQ & 85 & 88.5 & 80 & 83.3 & 6 & 7.4 & 17 & 21.0 \\
\hline AHQ/ALRQ & 4 & 4.2 & 4 & 4.2 & 19 & 23.5 & 26 & 32.1 \\
\hline ALRQ/VRQ & 2 & 2.1 & 2 & 2.1 & - & - & - & - \\
\hline ARR/ALRQ & - & - & - & - & 4 & 4.9 & 8 & 9.9 \\
\hline AFRQ/ALRQ & 4 & 4.2 & 9 & 9.4 & 10 & 12.3 & 3 & 3.7 \\
\hline AHQ/AFRQ & - & - & - & - & 8 & 9.9 & 1 & 1.2 \\
\hline ARR/AFRQ & - & - & - & - & 4 & 4.9 & - & - \\
\hline AFRQ/AFRQ & - & - & - & - & 5 & 6.2 & 1 & 1.2 \\
\hline Others $^{\mathrm{b}}-\mathrm{L}_{141}$ & 1 & 1.0 & 1 & 1.0 & 25 & 30.9 & 25 & 30.9 \\
\hline Others $^{\mathrm{b}}-\mathbf{F}_{141}$ & - & - & - & - & - & - & - & - \\
\hline Total & 96 & 100.0 & 96 & 100.0 & 81 & 100.0 & 81 & 100.0 \\
\hline
\end{tabular}

a AHQ, ARH, ARR, VRQ.

b AHQ/AHQ, AHQ/ARH, ARR/AHQ, ARR/ARR, ARH/VRQ.

In the present study, seven German sheep flocks were identified where more than a single atypical scrapie case was observed which may indicate an infectious origin of this disease. However, since these cohort cases to atypical scrapie cases were confined to larger flocks (more than 500 sheep), the possibility remains for their sporadic origin, if they occur at a frequency of 1:2000 or less in the overall sheep population. Nevertheless, two Nor98-affected sheep have also been observed in a small Irish flock of 18 adult sheep [32].

As in Norway [30] and Portugal [33], German atypical scrapie cases were found throughout the country, whereas classical scrapie was restricted to northern parts of Germany, with the exception of one case. This difference between the two scrapie types may also infer a spontaneous and ubiquitous character of atypical scrapie. However, four outbreaks of classical scrapie had been observed in the south of Germany (Baden-Württemberg) between 1985 and 1998 [26]. Although three of these affected flocks consisted of Suffolk sheep [26], a breed which is often imported from the United Kingdom to Germany, it is unclear if these outbreaks had been caused by the import of sheep affected with classical scrapie.

The absence of atypical scrapie cases in Schleswig-Holstein, although having the second largest sheep population of all 


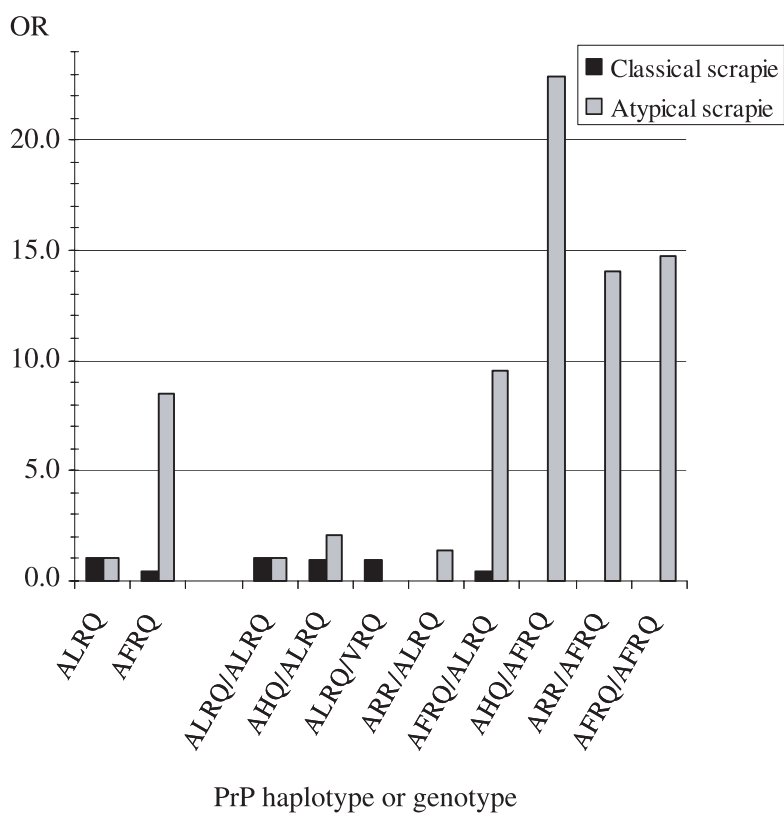

Figure 3. Odds ratios of the $\operatorname{PrP}$ haplotypes $A L_{141} R Q$ (ALRQ) and $A F_{141} R Q$ (AFRQ) and the resulting genotypes in genotype-matched (PrP codons 136, 154 and 171) pairs of scrapie-positive sheep and their flock mates with respect to the scrapie type. For each haplotype or genotype, the relative classical or atypical scrapie risk (odds ratio, OR) was calculated by dividing the quotient of its frequency $(\%)$ in scrapie-positive sheep and its frequency $(\%)$ in the flock mates by the quotient of the ALRQ or the ALRQ/ALRQ frequency (\%) in scrapie-positive sheep and in the flock mates.

German Federal states, may be due to the application of a TSE rapid test for small ruminants that has received the EU approval but does not detect atypical scrapie cases $[7,16]$.

Regarding the age of scrapie-affected sheep, it has to be kept in mind that a significant proportion of these animals were killed during the selective culling of a scrapie flock (particularly in classical scrapie cases) or were routinely slaughtered (particularly in atypical scrapie cases) and therefore did not develop clinical scrapie. Most German sheep diagnosed with classical scrapie were between 3 and 5 years of age at the time of death. Although the age of only a few classical scrapie-positive sheep was recorded, this result fits well with the usu- ally observed incubation times in classical scrapie, for which an infection of newborn lambs by their ewes is assumed [34]. On the contrary, the ages of sheep affected with atypical scrapie at the time of death were distributed over all age groups, with the majority of these sheep being older than five years of age. Furthermore, some of the sheep affected with atypical scrapie were exceptionally old with ages of up to 20 years (data not shown). In accordance with [30], we did not observe a significant influence of PrP haplotypes regarding codons 136, 154 and 171 or the codon 141 alleles on the age of sheep with atypical scrapie at the time of death. There may be other genetical or epidemiological factors (e.g. the route of infection or the age at infection) which influence the 
incubation time in atypical scrapie cases. Alternatively, atypical scrapie may occur spontaneously, particularly albeit not exclusively, in older animals.

Most scrapie-affected flocks consisted of crossbred sheep and except the Merinolandschaf breed no more than three purebred flocks of the same breed were affected by classical or atypical scrapie. Therefore, it was not possible to conclude an over- or underrepresentation of these breeds compared to all scrapie-affected flocks. No purebred Merinolandschaf flock was affected by classical scrapie, while 12 purebred flocks were affected with atypical scrapie. This is more likely due to the location of these flocks than to a selective resistance to classical scrapie and/or a susceptibility to atypical scrapie. The Merinolandschaf is the largest breed in Germany, most common in southern Germany and rarer in other parts of the country. Moreover, classical scrapie in a German Merinolandschaf had been diagnosed in 1998 [26].

Regarding the associations between $\operatorname{PrP}$ haplotypes and genotypes of codons 136, 154 and 171 with susceptibility to scrapie, the situation is relatively simple for German classical scrapie cases. While sheep carrying at least one ARR haplotype seem to be considerably more resistant to classical scrapie, the ARQ haplotype confers by far the highest risk in the German classical scrapie cases. However, this "resistance" of ARR carriers is not absolute as demonstrated by successful experimental BSEinfection of sheep with this genotype [22]. Moreover, one report exists on a sheep with the ARR/ARR genotype diagnosed with scrapie [25], which, however, has been received with scepticism. Furthermore, few classical scrapie-positive sheep carrying a single ARR allele and no VRQ allele have been identified in the EU-member states so far, e.g. in Belgium, France, Ireland and Spain [13] and in 1996 in a single German sheep [26].
Interestingly, the VRQ haplotype showed a low risk for classical scrapie in Germany. Generally, the VRQ haplotype is believed to confer the highest susceptibility to classical scrapie in populations where it is present (e.g. $[1,8,12])$. Nevertheless, the German situation resembles that of Italy, where classical scrapiepositive sheep from 2002-2004 did not carry the VRQ haplotype although VRQ has been found at a low frequency in random samples [13]. Finally, in the present study the $F_{141}$ allele occurred in four classical scrapie-positive sheep. This is in contrast to the findings of [36] who did not identify the $\mathrm{F}_{141}$ allele in Norwegian classical scrapie-positive sheep. There was no evidence that the polymorphism at codon 141 has an influence on the susceptibility for classical scrapie.

The molecular genetics of atypical scrapie seem to be more complex. The notably high risk values for the AFRQ and AHQ haplotypes and the genotypes including AFRQ (especially the AFRQ/AHQ genotype) are striking and strongly confirm former results $[3,30]$. But it should be kept in mind that in the present study nearly $50 \%$ of the sheep affected with atypical scrapie still carried the ALRQ and/or the ARR haplotype.

As in this study, a relatively low number of atypical scrapie-positive sheep with the ALRQ/ALRQ genotype compared to an about threefold higher frequency in the flock mates has also been observed by Moum et al. [30]. This supports a low atypical scrapie risk of this genotype compared to other affected genotypes, contradicting strongly with the situation in (German) classical scrapie cases. Nevertheless, ALRQ/ALRQ does not seem to confer an absolute resistance against atypical scrapie.

In contrast to $[3,30]$, the VRQ haplotype occurred with atypical scrapie in the present study, although it has only been found in one single sheep. 
In atypical scrapie, ARK was the only PrP haplotype that occurred in flock mates (but within one flock only) and was not found at least in one scrapie-positive sheep.

The current eradication strategy by the European Union (EU) for TSE in sheep requires a selection for the ARR haplotype (Commission Regulation 1492/2004 and Commission Decision 2003/100/EC), which is countered by our observation that one third of the atypical scrapie cases carried at least one ARR haplotype and $6.8 \%$ were homozygous for ARR. Although sheep with the AFRQ and/or the AHQ haplotype are even more susceptible to atypical scrapie than sheep carrying ARR, it can be foreseen that the PrP haplotypes AFRQ and AHQ both will vanish from the European sheep population through selection for ARR. Even though somewhat more classical $(n=120)$ than atypical $(n=97)$ scrapie-positive sheep were identified in Germany from January 2002 to February 2006, more than $80 \%$ of the scrapie-affected flocks were affected by atypical scrapie. Particularly with regards to this situation and the likeliness that atypical scrapie will not be eliminated by the implementation of the actual EUregulated breeding schemes for scrapie resistance, continued research on the aetiology, epidemiology, zoonotic potential and on genetic susceptibility factors of atypical scrapie is necessary.

\section{ACKNOWLEDGEMENTS}

The authors thank the German Veterinary Authorities and the sheep holders for their excellent support in the characterization of scrapie-affected sheep flocks. This work was financially supported by the Federal Ministry for Consumer Protection, Nutrition and Agriculture (research projects 02HS024 and 04HS059) and by the EU commission 'Network of Excellence' Neuroprion.

\section{REFERENCES}

[1] Baylis M., Chihota C., Stevenson E., Goldmann W., Smith A., Sivam K., Tongue S., Gravenor M.B., Risk of scrapie in British sheep of different prion protein genotype, J. Gen. Virol. (2004) 85:2735-2740.

[2] Belt P.B., Muileman I.H., Schreuder B.E., Bos-de Ruijter J., Gielkens A.L., Smits M.A., Identification of five allelic variants of the sheep PrP gene and their association with natural scrapie, J. Gen. Virol. (1995) 76:509-517.

[3] Benestad S.L., Sarradin P., Thu B., Schönheit J., Tranulis M.A., Bratberg B., Cases of scrapie with unusual features in Norway and designation of a new type, Nor98, Vet. Rec. (2003) 153:202-208.

[4] Bossers A., Schreuder B.E., Muileman I.H., Belt P.B., Smits M.A., PrP genotype contributes to determining survival times of sheep with natural scrapie, J. Gen. Virol. (1996) 77:2669-2673.

[5] Bruce M.E., Will R.G., Ironside J.W., McConnell I., Drummond D., Suttie A., McCardle L., Chree A., Hope J., Birkett C., Cousens S., Fraser H., Bostock C.J., Transmissions to mice indicate that 'new variant' CJD is caused by the BSE agent, Nature (1997) 389:498-501.

[6] Buschmann A., Lühken G., Schultz J., Erhardt G., Groschup M.H., Neuronal accumulation of abnormal prion protein in sheep carrying a scrapie-resistant genotype (PrPARR/ARR), J. Gen. Virol. (2004) 85:2727-2733.

[7] Buschmann A., Biacabe A.G., Ziegler U., Bencsik A., Madec J.Y., Erhardt G., Lühken G., Baron T., Groschup M.H., Atypical scrapie cases in Germany and France are identified by discrepant reaction patterns in BSE rapid tests, J. Virol. Methods (2004) 117:27-36.

[8] Dawson M., Hoinville L.J., Hosie B.D., Hunter N., Guidance on the use of PrP genotyping as an aid to the control of clinical scrapie. Scrapie Information Group, Vet. Rec. (1998) 142:623-625.

[9] De Bosschere H., Roels S., Benestad S.L., Vanopdenbosch E., Scrapie case similar to Nor98 diagnosed in Belgium via active surveillance, Vet. Rec. (2004) 155:707-708. 
[10] De Bosschere H., Roels S., Dechamps P., Vanopdenbosch E., TSE detected in a Belgian ARR-homozygous sheep via active surveillance, Vet. J. (2005) doi:10.1016/j.tvj1.2005.07.014.

[11] Detwiler L.A., Baylis M., The epidemiology of scrapie, Rev. Sci. Tech. (2003) 22:121-143.

[12] Díaz C., Vitezica Z.G., Rupp R., Andréoletti O., Elsen J.M., Polygenic variation and transmission factors involved in the resistance/susceptibility to scrapie in a Romanov flock, J. Gen. Virol. (2005) 86:849-857.

[13] Directorate-General E.C.H.C.P., Report on the monitoring and testing of ruminants for the presence of transmissible spongiform encephalopathy (TSE) in the EU in 2004, Brussels, 2005.

[14] Dubois M.A., Sabatier P., Durand B., Calavas D., Ducrot C., Chalvet-Monfray K., Multiplicative genetic effects in scrapie disease susceptibility, C. R. Biol. (2002) 325:565-570.

[15] Eddy R.G., Origin of BSE, Vet. Rec. (1995) 137:648.

[16] EFSA, Scientific report of the Euopean Food Safety Authority on the evaluation of rapid post mortem tests intended for small ruminants, question No. EFSA-Q-2003-084, adopted at 17 May 2005.

[17] Goldmann W., Hunter N., Smith G., Foster J., Hope J., PrP genotype and agent effects in scrapie: change in allelic interaction with different isolates of agent in sheep, a natural host of scrapie, J. Gen. Virol. (1994) 75:989-995.

[18] Goldmann W., Baylis M., Chihota C., Stevenson E., Hunter N., Frequencies of PrP gene haplotypes in British sheep flocks and the implications for breeding programmes, J. Appl. Microbiol. (2005) 98:1294-1302.

[19] Gretzschel A., Buschmann A., Eiden M., Ziegler U., Lühken G., Erhardt G., Groschup M.H., Strain typing of German transmissible spongiform encephalopathies field cases in small ruminants by biochemical methods, J. Vet. Med. B Infect. Dis. Vet. Public Health (2005) 52:55-63.

[20] Gretzschel A., Buschmann A., Langeveld J., Groschup M.H., Immunological characterization of abnormal prion protein from atypical scrapie cases in sheep using a panel of monoclonal antibodies, J. Gen. Virol. (2006) $87: 3715-3722$.

[21] Hill A.F., Desbruslais M., Joiner S., Sidle K.C., Gowland I., Collinge J., Doey L.J., Lantos P., The same prion strain causes vCJD and BSE, Nature (1997) 389:448-450, 526.

[22] Houston F., Goldmann W., Chong A., Jeffrey M., Gonzalez L., Foster J., Parnham D., Hunter N., Prion diseases: BSE in sheep bred for resistance to infection, Nature (2003) 423:498.

[23] Hunter N., Foster J.D., Goldmann W., Stear M.J., Hope J., Bostock C., Natural scrapie in a closed flock of Cheviot sheep occurs only in specific PrP genotypes, Arch. Virol. (1996) 141:809-824.

[24] Hunter N., PrP genetics in sheep and the applications for scrapie and BSE, Trends Microbiol. (1997) 5:331-334.

[25] Ikeda T., Horiuchi M., Ishiguro N., Muramatsu Y., Kai-Uwe G.D., Shinagawa M., Amino acid polymorphisms of PrP with reference to onset of scrapie in Suffolk and Corriedale sheep in Japan, J. Gen. Virol. (1995) 76:2577-2581.

[26] Junghans F., Teufel B., Buschmann A., Steng G., Groschup M.H., Genotyping of German sheep with respect to scrapie susceptibility, Vet. Rec. (1998) 143:340-341.

[27] Le Dur A., Beringue V., Andreolétti O., Reine F., Lai T.L., Baron T., Bratberg B., Vilotte J.L., Sarradin P., Benestad S.L., Laude H., A newly identified type of scrapie agent can naturally infect sheep with resistant PrP genotypes, Proc. Natl. Acad. Sci. USA (2005) 102:16031-16036.

[28] Lühken G., Buschmann A., Groschup M.H., Erhardt G., Prion protein allele A136 H154Q171 is associated with high susceptibility to scrapie in purebred and crossbred German Merinoland sheep, Arch. Virol. (2004) 149:1571-1580.

[29] Madec J.Y., Simon S., Lezmi S., Bencsik A., Grassi J., Baron T., Abnormal prion protein in genetically resistant sheep from a scrapie-infected flock, J. Gen. Virol. (2004) 85:3483-3486.

[30] Moum T., Olsaker I., Hopp P., Moldal T., Valheim M., Moum T., Benestad S.L., Polymorphisms at codons 141 and 154 in the ovine prion protein gene are associated with 
scrapie Nor98 cases, J. Gen. Virol. (2005) $86: 231-235$.

[31] OIE, Manual of diagnostic tests and vaccines for terrestrial animals (mammals, birds and bees) 2, 2004, pp. 642-653.

[32] Onnasch H., Gunn H.M., Bradshaw B.J., Benestad S.L., Bassett H.F., Two Irish cases of scrapie resembling Nor98, Vet. Rec. (2004) 155:636-637.

[33] Orge L., Galo A., Machado C., Lima C., Ochoa C., Silva J., Ramos M., Simas J.P., Identification of putative atypical scrapie in sheep in Portugal, J. Gen. Virol. (2004) 85:3487-3491.

[34] Pattison I.H., Hoare M.N., Jebbett J.N., Watson W.A., Spread of scrapie to sheep and goats by oral dosing with foetal membranes from scrapie-affected sheep, Vet. Rec. (1972) 90:465-468.

[35] Sigurdarson S., Epidemiology of scrapie in Iceland and experience with control measures, in: Bradley R. et al. (Eds.), Sub-Acute Spongiform Encephalopathies, Kluwer Academic Publishers, Dordrecht, 1991, pp. 233-242.

[36] Tranulis M.A., Osland A., Bratberg B., Ulvund M.J., Prion protein gene polymorphisms in sheep with natural scrapie and healthy controls in Norway, J. Gen. Virol. (1999) 80:1073-1077.

[37] Vitezica Z.G., Elsen J.M., Rupp R., Diaz C., Using genotype probabilities in survival analysis: a scrapie case, Genet. Sel. Evol. (2005) 37:403-415.

[38] Wilesmith J.W., Wells G.A., Cranwell M.P., Ryan J.B., Bovine spongiform encephalopathy: epidemiological studies, Vet. Rec. (1988) 123:638-644.

[39] Wilesmith J.W., Ryan J.B., Atkinson M.J., Bovine spongiform encephalopathy: epidemiological studies on the origin, Vet. Rec. (1991) 128:199-203. 